Base (IDB). The IDB comprises two datasets: the Full Data Set (FDS) and Minimum Data Set (MDS). Although the MDS collects less detail than the FDS; it is simpler for countries to adopt, and still sufficient to allow enumeration of injuries in key areas such as the home, leisure, work, road, falls, sports, and self-harm. Training, guides and rigorous quality checks, ensure consistency across participating countries.

Results To date, 26 countries have submitted 7,170,069 ED records (years 2009-2014) to the IDB in MDS format, and 20 countries have provided reference population data, enabling the calculation of incidence rates. As an exemplar, in 2013, incidence rates for all injuries varied between $11.43 \%$ in Luxembourg to $3.98 \%$ in Finland; the reasons behind these variations will be discussed at the conference. The MDS has provided a valuable source of data for several organisations across Europe, and can be accessed via several channels, including an online tool. The MDS strives to contribute data to the "European Core Health Indicators" (ECHI), "home, leisure and school accidents" (ECHI29) indicator.

Conclusions The MDS provides Europe with a valuable source of comparable injury data. Work is currently underway to ensure the MDS data is as valid and representative as possible.

\section{A EUROPEAN CORE HEALTH INDICTOR FOR HOME AND LEISURE INJURIES (ECHI-29)}

${ }^{1}$ Rupert Kisser, ${ }^{2}$ Samantha Turner, ${ }^{2,3}$ Ronan A Lyons, ${ }^{4}$ Wim Rogmans, ${ }^{5}$ Bjarne Larsen, ${ }^{6}$ Huib Valkenberg, ${ }^{7}$ Dritan Bejko, ${ }^{8}$ Robert Bauer, ${ }^{8}$ Monica Steiner. ${ }^{1}$ Eurosafe, Austria; ${ }^{2}$ Farr Institute Swansea University, Medical School, UK; ${ }^{3}$ Public Health Wales NHS Trust, UK; ${ }^{4}$ Eurosafe, The Netherlands; ${ }^{5}$ National Institute of Public Health, Denmark; ${ }^{6}$ Consumer Safety Institute, The Netherlands; ${ }^{7}$ Centre d'Etudes en Santé Publique, Luxembourg; ${ }^{8}$ Austrian Road Safety Board, Austria

\subsection{6/injuryprev-2016-042156.221}

Background Within the framework of the EU health information system, a European Core Health Indictor (ECHI) is foreseen on home and leisure, sport and school injuries (ECHI-29). However in 2010, there were no reasonably comparable injury data available for these areas, but emergency department (ED) records offered a most valuable source of information.

Methods Based on previous experiences, the Joint Action on Monitoring Injuries in Europe (JAMIE) project (2010-2013) has developed a comprehensive Minimum Data Set (MDS), which can be recorded in EDs without adding noteworthy burden to staff and patients. MDS contains diagnoses and allows the enumeration of injuries in key areas as road, workplace, home and leisure, sport, school, work place, self-harm and assault. MDS data can be recorded directly or extracted from other datasets as ICD-10 or IDB-FDS (Full Data Set), which is used for recording injuries related to consumer products.

Results During 2011-2013, 26 countries have submitted national MDS data sets to the European Injury Data Base (IDB) in MDS format, and 20 countries have provided reference population data, enabling the calculation of ECHI29. There are about 31 million home, leisure, sport and school injuries each year in the EU-28, which is $76 \%$ of all injuries treated in EDs. 700.000 injuries occur at school, 5.9 million during sporting, and 24.7 million at home or during other leisure time activities. The average incidence rate was $6.1 \%$, with a variation between $4.9 \%$ in Portugal and $8.9 \%$ in Luxembourg.

Conclusions MDS records from emergency departments can be the source for meaningful injury statistics and allow a differentiation by settings, age-groups and type of injuries. However, considerable variations between countries indicate that improvement of national methods is needed in order to increase crosscountry comparability.

\section{COMBINING SURVEY AND REGISTER BASED DATA TO ESTIMATE BURDEN OF INJURIES AMONG ADOLESCENTS}

${ }^{1}$ Dritan Bejko, ${ }^{2}$ Rupert Kisser, ${ }^{3,4}$ Ronan A Lyons, ${ }^{5}$ Yossi Harel-Fisch, ${ }^{6}$ Bjarne Larsen, ${ }^{7}$ Wim Rogmans, ${ }^{2}$ Samantha Turner, ${ }^{8}$ Robert Bauer, ${ }^{9}$ Gabrielle Ellsessaer, ${ }^{10}$ Huib Valkenberg. ${ }^{1}$ Luxembourg Institute of Health, Luxembourg; ${ }^{2}$ Eurosafe, Austria; ${ }^{3}$ Farr Institute Swansea University, Medical School, UK; ${ }^{4}$ Public Health Wales NHS Trust, UK; ${ }^{5}$ Bar Ilan University, Israe; ${ }^{6}$ National Institute of Public Health, Denmark; ${ }^{7}$ Eurosafe, The Netherlands; ${ }^{8}$ Austrian Road Safety Board, Austria; ${ }^{9}$ Landesamt Brandenburg Für Umwelt, Gesundheit Und Verbraucherschutz, Germany; ${ }^{10}$ Consumer Safety Institute, The Netherlands

\subsection{6/injuryprev-2016-042156.222}

Background Children and adolescents have the highest risk of injury. Emergency department (ED) data provide the best opportunity for estimating the burden of hospital treated injuries. Survey based data remains the only source of information for out of hospital medically treated injuries in many countries. The purpose of this study was to describe the incidence of selected injuries among adolescents in 16 European countries using surveybased and registry based methods.

Methods Survey based data were collected during the 2013/2014 wave of the Health Behaviour in School Aged Children (HBSC) study in 42 countries. Children reported the number of medically treated injuries for the last 12 months, the severity of the most serious injury as well as the place of occurrence and the activity when injured. The Registry based data supplied to the European Injury Data Base (IDB) for the period 2013-2014 provided detailed information about causes and circumstances of ED treated injuries for the same period and age group.

Results Detailed analyses were possible using IDB data from 16 countries and HBSC data from 42 countries. Both sources confirm an inter-country variability for burden of injuries among adolescents. Due to different methodologies in defining an injury case, comparison is not always possible between registry based and survey based data collection.

Conclusions Survey based and register based data can be used as complementary sources of information to have a full picture of injury burden among adolescents in many European countries.

\section{ARE REGISTER-BASED DATA BETTER THAN SURVEYS IN ESTIMATING BURDEN OF INJURIES AMONG ADULTS? RESULTS FROM LUXEMBOURG}

${ }^{1}$ Dritan Bejko, ${ }^{1}$ Maria Ruiz-Castell, ${ }^{2,3}$ Ronan A Lyons, ${ }^{4}$ Rupert Kisser, ${ }^{5}$ Bjarne Larsen, ${ }^{6}$ Wim Rogmans, ${ }^{2}$ Samantha Turner, ${ }^{7}$ Robert Bauer, ${ }^{8}$ Gabrielle Ellsessaer, ${ }^{9}$ Huib Valkenberg. 'Luxembourg Institute of Health, Luxembourg; ${ }^{2}$ Farr Institute Swansea University, Medical School, UK; ${ }^{3}$ Public Health Wales NHS Trust, UK; ${ }^{4}$ Eurosafe, Austria; ${ }^{5}$ National Institute of Public Health, Denmark; ${ }^{6}$ Eurosafe, The Netherlands; ${ }^{7}$ Austrian Road Safety Board, Austria; ${ }^{8}$ Landesamt Brandenburg Für Umwelt, Gesundheit Und Verbraucherschutz, Germany; ${ }^{9}$ Consumer Safety Institute, The Netherlands

\subsection{6/injuryprev-2016-042156.223}

Background Injury is a major cause of mortality and morbidity. The emergency department (ED) registry based data, provides a cost-effective way to estimate the burden of injuries. Previous studies in general population have suggested that survey based data collection is not efficient and suffers from recall or selection bias. The aim of this study was to compare the yearly incidence 